\title{
Rare Manifestations of Churg-Strauss Syndrome with Mediastinal and Hilar Lymphadenopathies: Report of an Autopsy Case
}

\author{
Woo Cheal Cho · Bharat Ramlal · Mary Fiel-Gan · Xianyuan Song \\ Department of Pathology and Laboratory Medicine, Hartford Hospital, Hartford, CT, USA
}

Churg-Strauss syndrome (CSS) is a rare systemic disorder that is classically characterized by asthma, tissue and blood eosinophilia, and necrotizing vasculitis along with a granulomatous response to eosinophilic necrosis in its full-blown form. It has a predilection for small- and medium-sized vessels of the lungs, but can virtually affect any organ system, including the heart, skin, gastrointestinal tract, and nervous system. Involvement of the lymph node in CSS, however, is still rare with only a few cases reported in the English literature. ${ }^{1-6}$ Furthermore, the classic histologic features of CSS, particularly necrotizing vasculitis, are infrequently captured in the modern biopsy or autopsy specimens due to the widespread use of steroids. ${ }^{7}$ Here, we report the autopsy findings of a 69-year-old man with CSS involving mediastinal and hilar lymph nodes and discuss the histologic features of partially treated CSS.

Formal written informed consent was not required with a waiver by the appropriate institutional review board and/or national research ethics committee (FWA00021932).

\section{CASE REPORT}

A 69-year-old man presented to the Emergency Department after a witnessed cardiac arrest and subsequently died shortly thereafter. On autopsy, ruptured abdominal aortic aneurysm $(7.0 \mathrm{~cm}$ in greatest diameter) with a $2.0-\mathrm{cm}$ linear tear, moder-

\section{Corresponding Author}

Woo Cheal Cho, MD

Department of Pathology and Laboratory Medicine, Hartford Hospital, 80 Seymour

Street, Hartford, CT 06102-5037, USA

Tel: +1-860-972-2488, Fax: +1-860-545-2204

E-mail: woocheal.cho@hhchealth.org

Received: September 6, 2017 Revised: December 13, 2017

Accepted: December 13, 2017 ate-severe atherosclerosis of abdominal aorta, cardiomegaly (500 g) with biventricular hypertrophy, congestive hepatomegaly $(2,050 \mathrm{~g})$, and multiple bilateral pulmonary nodules (measuring up to $1.5 \mathrm{~cm}$ ) with significant mediastinal and hilar lymphadenopathies (Fig. 1A) were grossly identified. Histologically, the lung nodules showed multifocal necrotizing granulomas with surrounding tissues infiltrated by lymphocytes and rare scattered eosinophils (Fig. 1B). Extravascular eosinophilic infiltration with associated granulomatous inflammation (Fig. 1C) but without overt necrotizing vasculitis, as well as foci of mildmoderate peribronchial eosinophilic infiltration, was also noted. A few small-sized arteries showed prominent necrotizing granulomatous inflammation focally involving the vessel wall (Fig. 2A, B). Occasionally, rare eosinophils with partially degenerated nuclei were found within the center of the necrosis (Fig. 2C). Most vessels involved by granulomatous inflammation did not reveal an overt necrotizing vasculitis, and viable eosinophils with intact nuclei were more readily identified within these granulomas (Fig. 2D, E). The mediastinal and hilar lymph nodes showed prominent necrotizing granulomas diffusely involving the nodal parenchyma; the center of the necrotizing granulomas displayed numerous necrotic or degenerated cells with acidophilic granules (Fig. 3A-C). In contrast, viable eosinophils with intact nuclei were more readily identified at the periphery or outside the necrotic zone (Fig. 3D). Special stains for acid-fast bacilli and fungal microorganisms were negative in sections of the lung nodules as well as the lymph nodes. The abdominal aorta, including the area of rupture, was extensively sectioned but revealed no evidence of vasculitis, granulomatous disease, or tissue eosinophilia. Additional clinical information obtained following the autopsy revealed that the patient had chronic asthma, sinus abnormalities, peripheral hypereosinophilia, and perinuclear 
anti-neutrophil cytoplasmic antibody on serology, confirming the diagnosis of CSS. Further review of the patient's history also revealed use of corticosteroids, which had recently been tapered and were switched to rituximab.

\section{DISCUSSION}

CSS is a rare systemic disorder with a reported annual incidence of 1-2 cases per million persons in the general population. ${ }^{8} \mathrm{It}$ was first described by Churg and Strauss ${ }^{9}$ in 1951 on 13 patients as "allergic granulomatosis, allergic angiitis, and periarteritis nodosa." The original cases described by Churg and Strauss ${ }^{9}$ shared a spectrum of many similar clinicopathologic features such as asthma, blood and tissue eosinophilia, necrotizing vascu- litis, and granulomatous response to eosinophilic necrosis. Recent studies, however, have suggested that this definition may indeed be too narrow, leading to the exclusion of certain cases that may not have such "classic" findings. ${ }^{7}$ The original 13 cases predated the use of exogenous steroids, thereby representing this entity in its untreated or full-blown form. In contrast, currently, steroids are widely used not only for asthmatics but also for the patients with suspected CSS. The widespread use of steroids in the modern era often leads to a confusing situation where the definitive or "classic" features of CSS, particularly eosinophilic necrotizing vasculitis, are absent. In addition, CSS has an early, "pre-vasculitic" or "prodromal" phase characterized by tissue infiltration of eosinophils without overt vasculitis, as well as postvasculitic phase (if successfully treated) in which dense organizing thrombi
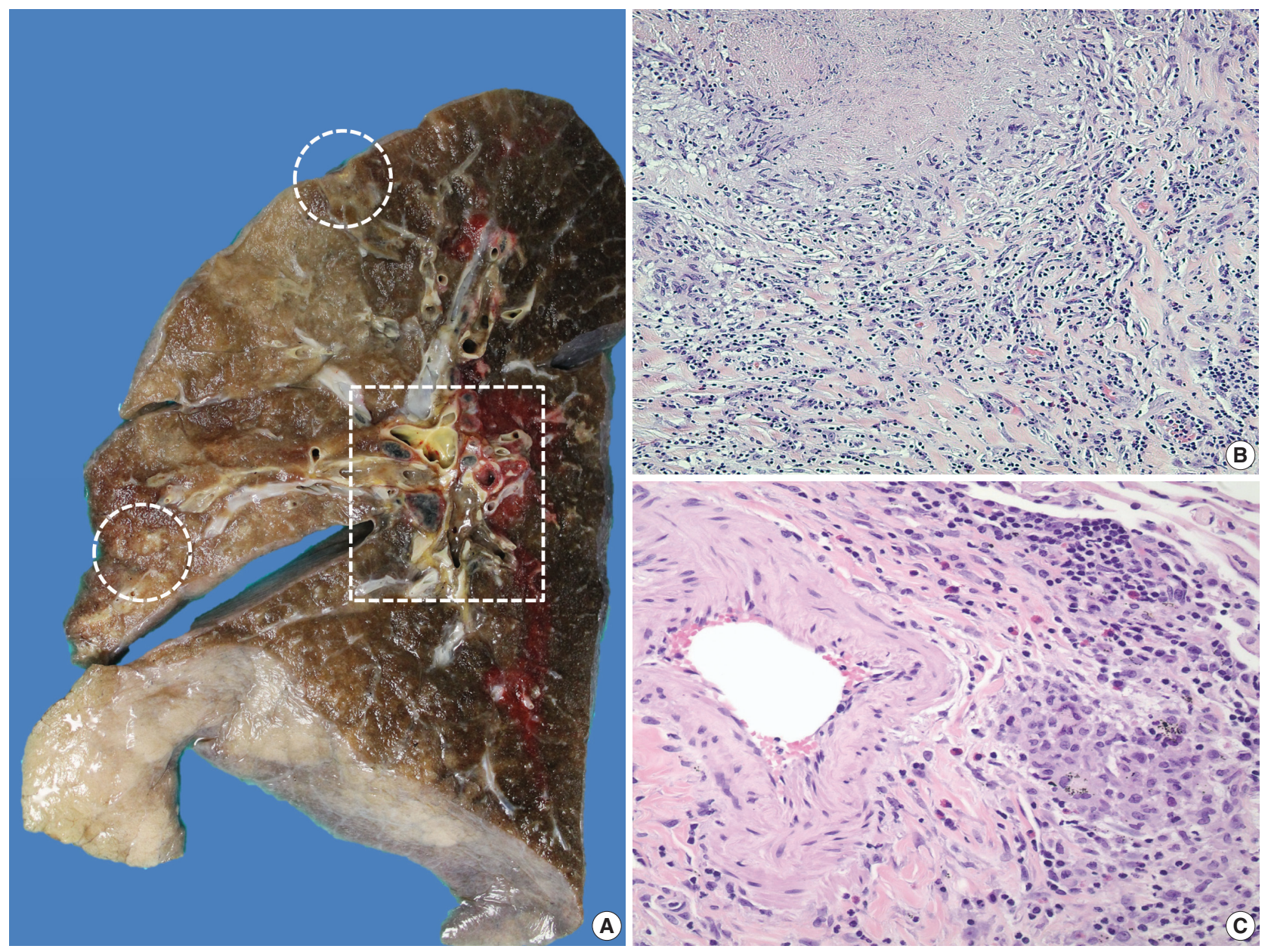

Fig. 1. Gross and histologic findings of pulmonary nodules. (A) The right lung (570 g) grossly displays multiple peripherally located palpable nodules (dashed circles), measuring up to $1.5 \mathrm{~cm}$ in greatest dimension. The nodules are tan, firm, and relatively well-circumscribed with an irregular border. Also noted are significant subcarinal (not shown) and hilar (dashed rectangle) lymphadenopathies with a mild-moderate anthracotic pigment deposition. (B) Histologically, the lung nodules display prominent necrotizing granulomatous inflammation with surrounding tissues infiltrated by lymphocytes and rare scattered eosinophils. (C) Extravascular eosinophilic infiltration with associated granulomatous inflammation without forming an overt necrotizing vasculitis is also noted. 

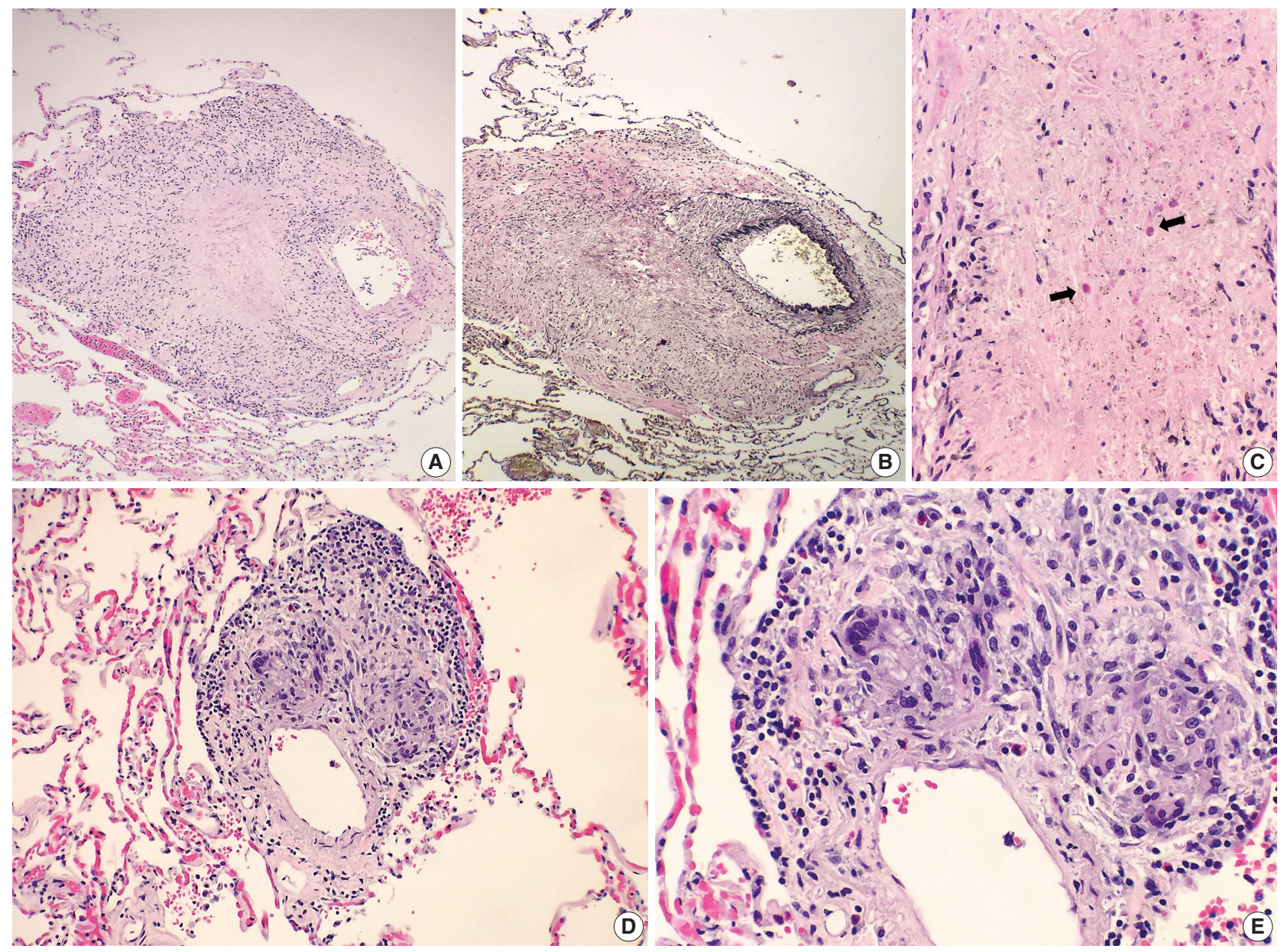

Fig. 2. Histologic spectrum of perivascular eosinophilic granulomatous inflammation. (A-C) A few small-sized arteries reveal a prominent necrotizing granulomatous inflammation focally involving the vessel wall (B, elastin stain). Occasionally, the center of the necrosis shows rare eosinophils with partially degenerated nuclei (arrows). (D, E) The majority of vessels involved by granulomatous inflammation do not reveal an overt necrotizing vasculitis. Viable eosinophils are more readily identified within these granulomas.

are seen within the vascular lumen in the absence of active vasculitis or eosinophils. Thus, the absence of eosinophilic necrotizing vasculitis should not preclude the diagnosis.

Currently, the diagnostic criteria proposed by the American College of Rheumatology (ACR $)^{10}$ is widely used with the broadest definition of CSS: (1) asthma, (2) paranasal sinus abnormalities, (3) eosinophilia greater than $10 \%$ on differential white blood cell count, (4) neuropathy (mono or poly), (5) pulmonary infiltrates, and (6) biopsy containing a blood vessel with extravascular eosinophils. The ACR criteria do not require pathologic evidence of vasculitis, and the presence of four or more of these six criteria have yielded a sensitivity of $85 \%$ and a specificity of $99.7 \% .^{10}$ In this present case, our patient fulfilled at least five out of six diagnostic criteria, and we believe our case represents a partially treated CSS.

In conclusion, our case illustrates rare manifestations of CSS and adds to the growing pool of case reports of CSS with lymphadenopathy; a finding which can raise the differential diagnosis that includes lymphoma and infection. Histologic descriptions of CSS involving lymph nodes is in the literature showing necrotizing granulomas and eosinophilic necrosis are scarce. Furthermore, an awareness of the histologic features of treated CSS is important, as strict adherence to the original diagnostic criteria may erroneously lead to an under- or misdiagnosis.

\section{ORCID}

Woo Cheal Cho: https://orcid.org/0000-0001-5867-1403

\section{Conflicts of Interest}

The authors declare that they have no potential conflicts of interest. 



Fig. 3. Histologic findings of lymph nodes. (A-D) The mediastinal and hilar lymph nodes display prominent necrotizing granulomas. Within the center of the necrosis are numerous necrotic or degenerating eosinophils without intact nuclei but predominantly with acidophilic granules (A-C). In contrast, the periphery of or outside the necrotic zone show more readily identifiable eosinophilic infiltrates (D).

\section{REFERENCES}

1. Casey M, Radel E, Ratech H. Lymph node manifestations of limited Churg-Strauss syndrome. J Pediatr Hematol Oncol 2000; 22: 468-71.

2. Choi JY, Kim JE, Choi IY, et al. Churg-Strauss syndrome that presented with mediastinal lymphadenopathy and calculous cholecystitis. Korean J Intern Med 2016; 31: 179-83.

3. Choi YH, Im JG, Han BK, Kim JH, Lee KY, Myoung NH. Thoracic manifestation of Churg-Strauss syndrome: radiologic and clinical findings. Chest 2000; 117: 117-24.

4. Churg A, Brallas M, Cronin SR, Churg J. Formes frustes of ChurgStrauss syndrome. Chest 1995; 108: 320-3.

5. Cualing H, Schroder L, Perme C. Allergic granulomatosis secondary to a limited form of Churg-Strauss syndrome. Arch Pathol Lab

\section{Med 2001; 125: 954-7.}

6. Lesens O, Hansmann Y, Nerson J, et al. Severe Churg-Strauss syndrome with mediastinal lymphadenopathy treated with interferon therapy. Eur J Intern Med 2002; 13: 458.

7. Churg A. Recent advances in the diagnosis of Churg-Strauss syndrome. Mod Pathol 2001; 14: 1284-93.

8. Watts RA, Carruthers DM, Scott DG. Epidemiology of systemic vasculitis: changing incidence or definition? Semin Arthritis Rheum 1995; 25: 28-34.

9. Churg J, Strauss L. Allergic granulomatosis, allergic angiitis, and periarteritis nodosa. Am J Pathol 1951; 27: 277-301.

10. Masi AT, Hunder GG, Lie JT, et al. The American College of Rheumatology 1990 criteria for the classification of Churg-Strauss syndrome (allergic granulomatosis and angiitis). Arthritis Rheum 1990; 33: 1094-100. 